\title{
Matriks Massa Neutrino Berat dari Model Seesaw
}

\author{
Agus Purwanto* \\ Laboratorium Fisika Teori dan Filsafat Alam (LaFTiFA), \\ Jurusan Fisika, FMIPA, Institut Teknologi Sepuluh Nopember, \\ Kampus ITS Sukolilo, Surabaya 60111
}

\begin{abstract}
Intisari
Dengan asumsi simetri lepton-quark dan menggunakan data eksperimen osilasi neutrino, struktur matriks massa neutrino berat dikaji dalam beberapa kasus. Hasilnya adalah dua bentuk dominan. Pertama, bentuk diagonal yang terkait dengan skala unifikasi dan di atasnya. Kedua, bentuk off-diagonal yang terkait dengan skala antara.

KATA KUNCI: osilasi neutrino, matrik bauran dan model seesaw
\end{abstract}

\section{PENDAHULUAN}

Di dalam Model Standar (MS) neutrino tidak bermassa karena neutrino hanya muncul dengan chiralitas kiri (lefthanded) $\nu_{L}$ sehingga suku massa Dirac tidak dapat dibangun. Selain itu, suku massa Majorana bagi $\nu_{L}$ tidak dapat dibangun hanya dengan satu doublet Higgs $\phi$ setelah perusakan simetri spontan. Tetapi eksperimen-eksperimen SuperKamiokande, K2K, SNO dan KamLAND [1] memberi bukti kuat bahwa neutrino bermassa meskipun sangat kecil. Dengan demikian perluasan terhadap MS perlu dilakukan untuk menampung kehadiran massa neutrino tersebut.

Mekanisme seesaw [2] merupakan mekanisme paling populer untuk membangkitkan massa kecil neutrino yakni dengan menambahkan neutrino singlet chiralitas kanan (righthanded) masif $\nu_{R}$ ke dalam MS sektor lepton. Penambahan neutrino kanan ini memberi massa Dirac $M_{D}$ bagi neutrino dengan mekanisme yang sama dengan sektor quark. Dengan demikian kita harapkan massa $M_{D}$ mempunyai orde yang sama dengan massa fermion lainnya.

Selain itu, penambahan $\nu_{R}$ juga memungkinkan kita mempunyai suku massa Majorana, $M_{N}$ yaitu $\frac{1}{2} \bar{\nu}_{R} M_{N} \nu_{R}^{c}$ dan nilainya tidak dibatasi oleh grup gauge dari MS. Artinya, kita mempunyai skala massa baru bagi teori diperluas yakni model seesaw ini. Inilah masalah pokok yang perlu difahami apakah skala baru ini terkait dengan fisika baru yakni grup gauge yang lebih besar dan pada energi berapa hal ini terjadi.

Massa masif neutrino kanan $M_{N}$ memberi massa kecil neutrino $M_{\nu}$ melalui mekanisme seesaw

$$
M_{\nu}=M_{D} M_{N}^{-1} M_{D}^{T}
$$

Massa efektif neutrino $M_{\nu}$ ini dapat ditentukan dari data eksperimen osilasi neutrino. Dengan demikian kita harus menentukan $M_{D}$ dan $M_{N}$.

Massa Dirac dari quark dan lepton bermuatan memperlihatkan struktur hirarki yang teratur, $m_{d}<<m_{s}<<m_{b}$ untuk massa quark-down $M_{d}, m_{u}<<m_{c}<<m_{t}$ untuk quark-up

*E-MAIL: purwanto@physics.its.ac.id
$M_{u}$ dan $m_{e}<<m_{\mu}<<m_{\tau}$ untuk lepton bermuatan $M_{\ell}$. Karena $M_{\ell} \approx M_{d}$ [3] maka adalah hal yang wajar kita menduga atau berharap bahwa $M_{D} \approx M_{u}$. Inilah yang diusulkan oleh GUT dan dikenal sebagai simetri lepton-quark yang akan digunakan untuk analisa di dalam makalah ini.

Simetri lepton-quark mengusulkan

$$
M_{D} \approx \frac{m_{\tau}}{m_{b}}\left(\begin{array}{ccc}
m_{u} & 0 & 0 \\
0 & m_{c} & 0 \\
0 & 0 & m_{t}
\end{array}\right)
$$

Bentuk diagonal ini berasal dari kenyataan bahwa bauran dalam sektor Dirac serupa dengan bauran kecil dalam sektor quark [4], dan faktor $m_{\tau} / m_{b} \equiv k$ yang merambat (running) dari skala unifikasi yang mana dalam skala ini $m_{b}=$ $m_{\tau}$ [5]. Massa Dirac neutrino mempunyai nilai $M_{D} \approx$ $\operatorname{diag}(0,001 ; 0,3 ; 100) \mathrm{GeV}[6]$.

Di dalam artikel ini akan ditentukan matriks massa neutrino berat dan mengestimasi skala baru melalui kebalikan pers.(1)

$$
M_{N}=M_{D}^{T} M_{\nu}^{-1} M_{D}
$$

dengan menggunakan data eksperimen massa dan bauran (mixing) neutrino serta asumsi simetri quark-lepton.

Di bagian II diuraikan matriks bauran sektor lepton, data massa dan sudut bauran dari osilasi neutrino matahari dan atmosfer. Bagian III membahas matriks massa neutrino berat dalam kasus hirarki normal,hirarki terbalik bagi massa Dirac neutrino dengan sudut bauran maksimal tunggal maupun bimaksimal. Akhirnya diberikan diskusi dan kesimpulan pada bagian IV. 


\section{MASSA NEUTRINO DAN MATRIKS BAURAN LEPTONIK}

Keadaan eigen flavor $\nu_{\ell}$ dan keadaan eigen massa $\nu_{i}$ dihubungkan oleh matriks bauran (mixing matrix) uniter $U$

$$
\begin{aligned}
\left(\begin{array}{c}
\nu_{e} \\
\nu_{\mu} \\
\nu_{\tau}
\end{array}\right) & =U\left(\begin{array}{c}
\nu_{1} \\
\nu_{2} \\
\nu_{3}
\end{array}\right) \\
& \equiv\left(\begin{array}{lll}
U_{e 1} & U_{e 2} & U_{e 3} \\
U_{\mu 1} & U_{\mu 2} & U_{\mu 3} \\
U_{\tau 1} & U_{\tau 2} & U_{\tau 3}
\end{array}\right)\left(\begin{array}{l}
\nu_{1} \\
\nu_{2} \\
\nu_{3}
\end{array}\right)
\end{aligned}
$$

Matriks bauran sektor lepton ini dikenal sebagai matriks bauran Pontecorvo-Maki-Nakagawa-Sakata (PMNS)[7].

Hasil eksperimen osilasi neutrino matahari memberi tiga solusi bagi masalah neutrino matahari yakni sudut bauran kecil (small mixing angle, SMA) MSW (Mikheyev-SmirnovWolfstein), sudut bauran besar (large mixing angle, LMA) MSW dan osilasi vakum (VO). Orde besaran bagi neutrino matahari $\Delta m_{\odot}^{2}[8]$

$$
\begin{aligned}
& \Delta m_{\odot}^{2} \approx 10^{-6} \mathrm{eV}^{2}(\text { SMA) } \\
& \Delta m_{\odot}^{2} \approx 10^{-5} \mathrm{eV}^{2} \text { (LMA) } \\
& \Delta m_{\odot}^{2} \approx 10^{-10} \mathrm{eV}^{2} \text { (VO) }
\end{aligned}
$$

Sedangkan osilasi atmosferik memberikan

$$
\Delta m_{a t m}^{2} \approx 10^{-3} e V^{2}
$$

Data-data di atas jelas memberikan $\Delta m_{\odot}^{2}<<\Delta m_{a t m}^{2}$. Secara teoritis hasil pengamatan massa kuadrat di atas terkait dengan selisih massa eigen kuadrat

$$
\begin{aligned}
\Delta m_{\odot}^{2} & =m_{2}^{2}-m_{1}^{2} \\
\Delta m_{\text {atm }}^{2} & =m_{3}^{2}-m_{2}^{2}, m_{3}^{2}-m_{1}^{2}
\end{aligned}
$$

Karena itu, dengan mengambil $m_{3}>0$ data di atas memberi hirarki massa normal

$$
\left|m_{1}\right|<\left|m_{2}\right|<<m_{3}
$$

atau hirarki

$$
\left|m_{1}\right| \approx\left|m_{2}\right|>>m_{3}
$$

yang disebut hirarki terbalik.

Matriks bauran di dalam pers.(4) terdiri dari tiga matriks rotasi terhadap masing-masing sumbu, yakni sumbu satu $U(23)$, dua $U(13)$ dan sumbu tiga $U(12)$,

$$
\begin{aligned}
& U(23)=\left(\begin{array}{ccc}
1 & 0 & 0 \\
0 & \cos \theta_{23} & \sin \theta_{23} \\
0 & -\sin \theta_{23} & \cos \theta_{23}
\end{array}\right) \\
& U(13)=\left(\begin{array}{ccc}
\cos \theta_{13} & 0 & \sin \theta_{13} \\
0 & 1 & 0 \\
-\sin \theta_{13} & 0 & \cos \theta_{13}
\end{array}\right) \\
& U(12)=\left(\begin{array}{ccc}
\cos \theta_{12} & \sin \theta_{12} & 0 \\
\sin \theta_{12} & \cos \theta_{12} & 0 \\
0 & 0 & 1
\end{array}\right)
\end{aligned}
$$

Matriks bauran PMNS diberikan oleh [9]

$$
U=U(23) U(13) U(12)
$$

Lebih lanjut, eksperimen reaktor CHOOZ [10] memberikan sudut $\theta_{13}$ kecil sekali, misalkan $\theta_{13} \rightarrow \epsilon$ maka

$$
U(13)=\left(\begin{array}{ccc}
1 & 0 & \epsilon \\
0 & 1 & 0 \\
-\epsilon & 0 & 1
\end{array}\right)
$$

Sedangkan kolaborasi SuperKamiokande memberikan bauran maksimal bagi neutrino atmosferik, $\theta_{23} \approx \pi / 4$ [11],

$$
U(23)=\left(\begin{array}{ccc}
1 & 0 & 0 \\
0 & \frac{1}{\sqrt{2}} & \frac{1}{\sqrt{2}} \\
0 & -\frac{1}{\sqrt{2}} & \frac{1}{\sqrt{2}}
\end{array}\right)
$$

Tuliskan $\theta_{12}$ sebagai $\theta$ maka matriks PMNS menjadi

$$
U=\left(\begin{array}{ccc}
\cos \theta & \sin \theta & \epsilon \\
-\frac{\sin \theta+\epsilon \cos \theta}{\sqrt{2}} & \frac{\cos \theta-\epsilon \sin \theta}{\sqrt{2}} & \frac{1}{\sqrt{2}} \\
\frac{\sin \theta-\epsilon \cos \theta}{\sqrt{2}} & -\frac{\cos \theta+\epsilon \sin \theta}{\sqrt{2}} & \frac{1}{\sqrt{2}}
\end{array}\right)
$$

\section{MATRIKS MASSA NEUTRINO}

Perhatikan kembali pers.(1) dan (3), karena $M_{N}$ simetrik maka $M_{\nu}$ juga. Karena itu, $M_{\nu}$ dapat didiagonalisasi menurut hubungan,

$$
U^{+} M_{\nu} U^{*}=\left(\begin{array}{ccc}
m_{1} & 0 & 0 \\
0 & m_{2} & 0 \\
0 & 0 & m_{3}
\end{array}\right)
$$

Dengan demikian, matriks massa neutrino diberikan oleh

$$
\begin{aligned}
M_{\nu} & =U\left(\begin{array}{ccc}
m_{1} & 0 & 0 \\
0 & m_{2} & 0 \\
0 & 0 & m_{3}
\end{array}\right) U^{T} \\
& =\left(\begin{array}{ccc}
\alpha+\epsilon^{2} m_{3} & \beta & \gamma \\
\beta & \rho+\frac{\epsilon^{2} \alpha}{2} & \sigma+\frac{\epsilon^{2} \alpha}{2} \\
\gamma & \sigma+\frac{\epsilon^{2} \alpha}{2} & \rho \\
&
\end{array}\right)
\end{aligned}
$$

dengan

$$
\begin{aligned}
\alpha & =\cos ^{2} \theta m_{1}+\sin ^{2} \theta m_{2} \\
\alpha^{\prime} & =\sin ^{2} \theta m_{1}+\cos ^{2} \theta m_{2} \\
\beta & =\frac{\epsilon\left(m_{3}-\alpha\right)+\sin \theta \cos \theta\left(m_{2}-m_{1}\right)}{\sqrt{2}} \\
\gamma & =\frac{\epsilon\left(m_{3}-\alpha\right)-\sin \theta \cos \theta\left(m_{2}-m_{1}\right)}{\sqrt{2}} \\
\sigma & =\frac{m_{3}-\alpha^{\prime}}{2} \\
\rho & =\frac{m_{3}+\alpha^{\prime}-2 \epsilon \sin \theta \cos \theta\left(m_{2}-m_{1}\right)}{2} \\
\rho^{\prime} & =\frac{m_{3}+\alpha^{\prime}+2 \epsilon \sin \theta \cos \theta\left(m_{2}-m_{1}\right)}{2}
\end{aligned}
$$


Tampak suku $\epsilon^{2}$ di dalam pers.(19) dapat diabaikan dan matriks massa $M_{\nu}$ menjadi [12]

$$
M_{\nu}=\left(\begin{array}{ccc}
\alpha & \beta & \gamma \\
\beta & \rho & \sigma \\
\gamma & \sigma & \rho
\end{array}\right)
$$

Invers matriks massa ini diberikan oleh

dengan

$$
\begin{aligned}
\Sigma= & \frac{1}{2}\left[\epsilon ^ { 2 } \left\{2 m_{1}^{2} m_{2}+m_{3}^{2}\left(m_{2}+m_{1}\right)-4 m_{1} m_{2} m_{3}\right.\right. \\
& \left.\left.+\left(m_{1}-m_{2}\right)\left(m_{1} m_{2}-m_{3}^{2}\right)\right\}-2 m_{1} m_{2} m_{3}\right] \\
\approx & -m_{1} m_{2} m_{3}
\end{aligned}
$$

Hubungan di atas kita gunakan untuk menentukan bentuk dominan (leading form) matriks neutrino dan skala baru massa Majorana terbesar $M_{N 33}$.

\section{A. Hirarki Normal}

Menggunakan hirarki (11) maka kuantitas-kuantitas (20) menjadi

$$
\begin{aligned}
\alpha & =\cos ^{2} \theta m_{1}+\sin ^{2} \theta m_{2} \\
\alpha^{\prime} & =\sin ^{2} \theta m_{1}+\cos ^{2} \theta m_{2} \\
\beta & =\frac{\epsilon m_{3}+\sin \theta \cos \theta\left(m_{2}-m_{1}\right)}{\sqrt{2}} \\
\gamma & =\frac{\epsilon m_{3}-\sin \theta \cos \theta\left(m_{2}-m_{1}\right)}{\sqrt{2}} \\
\sigma & =\frac{m_{3}}{2} \\
\rho & =\rho^{\prime}=\frac{m_{3}}{2}
\end{aligned}
$$

$$
M_{\nu}^{-1}=\frac{1}{\Sigma}\left(\begin{array}{ccc}
\sigma^{2}-\rho \rho & \beta \rho-\sigma \gamma & \rho \gamma-\beta \sigma \\
\beta \rho-\sigma \gamma & \gamma^{2}-\alpha \rho & \alpha \sigma-\beta \gamma \\
\gamma \rho-\beta \sigma & \alpha \sigma-\beta \gamma & \beta^{2}-\alpha \rho
\end{array}\right)
$$

dan matriks massa $M_{\nu}(21)$ menjadi

$$
M_{\nu}=\left(\begin{array}{ccc}
\alpha & \beta & \gamma \\
\beta & \frac{m_{3}}{2} & \frac{m_{3}}{2} \\
\gamma & \frac{m_{3}}{2} & \frac{m_{3}}{2}
\end{array}\right)
$$

Pers.(24) memperlihatkan bahwa $\alpha, \beta$, dan $\gamma$ merupakan besaran massa yang jauh lebih kecil dibanding $m_{3}$. Karena itu, bentuk dominan matriks $M_{\nu}$ berbentuk

$$
M_{\nu} \approx\left(\begin{array}{lll}
0 & 0 & 0 \\
0 & 1 & 1 \\
0 & 1 & 1
\end{array}\right)
$$

Selanjutnya, kita tentukan invers $M_{\nu}^{-1}$. Semua elemen matriks (22) dapat disubtitusi dengan kuantitas-kuantitas (24) dan didapatkan komponen $\left(M_{\nu}^{-1}\right)_{11}$ adalah nol. Komponen ini memang sangat kecil tetapi tidak perlu nol dan hal ini dapat diperoleh jika digunakan

$$
\begin{aligned}
\sigma & =\frac{m_{3}-\alpha}{2} \\
\rho & =\rho^{\prime}=\frac{m_{3}+\alpha^{\prime}}{2}
\end{aligned}
$$

Kuantitas (27) dan (24) mereduksi invers matriks (22) menjadi

$$
M_{\nu}^{-1} \approx \frac{1}{-\Sigma}\left(\begin{array}{ccc}
m_{3} \alpha, & \frac{m_{3}}{2}(\gamma-\beta) & \frac{m_{3}}{2}(\beta-\gamma) \\
\frac{m_{3}}{2}(\gamma-\beta) & \frac{m_{3}}{2} \alpha-\gamma^{2} & \beta \gamma-\frac{m_{3}}{2} \alpha \\
\frac{m_{3}}{2}(\beta-\gamma) & \beta \gamma-\frac{m_{3}}{2} \alpha & \frac{m_{3}}{2} \alpha-\beta^{2}
\end{array}\right)
$$

\section{Bauran Maksimal Tunggal}

sehingga

Pertama ambil kasus $\theta \approx 0$ maka besaran-besaran (24) menjadi

$$
\begin{aligned}
\alpha & =m_{1} \\
\alpha^{\prime} & =m_{2} \\
\beta & =\gamma=\frac{\epsilon m_{3}}{\sqrt{2}}
\end{aligned}
$$


dengan $x=\frac{m_{3}}{2}\left(m_{1}-\epsilon^{2} m_{3}\right)$. Matriks massa neutrino Majorana masif

$$
\begin{aligned}
M_{N}= & \frac{m_{\tau}^{2}}{-\Sigma m_{b}^{2}}\left(\begin{array}{ccc}
m_{u} & 0 & 0 \\
0 & m_{c} & 0 \\
0 & 0 & m_{t}
\end{array}\right)\left(\begin{array}{ccc}
m_{2} m_{3} & 0 & 0 \\
0 & x & -x \\
0 & -x & x
\end{array}\right) \\
& \times\left(\begin{array}{ccc}
m_{u} & 0 & 0 \\
0 & m_{c} & 0 \\
0 & & m_{t}
\end{array}\right) \\
= & \frac{k^{2}}{-\Sigma}\left(\begin{array}{ccc}
m_{2} m_{3} m_{u}^{2} & 0 & 0 \\
0 & x m_{c}^{2} & -x m_{c} m_{t} \\
0 & -x m_{c} m_{t} & x m_{t}^{2}
\end{array}\right)
\end{aligned}
$$

dengan $k=\frac{m_{\tau}}{m_{b}}$. Bentuk matriks massa ini memberi komponen terbesar

$$
M_{N 33} \approx \frac{k^{2} m_{\tau}^{2}}{2} \frac{\left(m_{1}-\epsilon^{2} m_{3}\right)}{m_{1} m_{2}}
$$

Pengandaian $\epsilon^{2} m_{3}<<m_{1}$ memberikan

$$
M_{N 33} \approx \frac{k^{2} m_{\tau}^{2}}{2 m_{2}}
$$

Sudut $\theta \approx 0$ merupakan kasus sudut baur kecil (SMA) dengan massa terkait

$$
m_{2} \leq \sqrt{\Delta m_{\odot}^{2}}=10^{-3} \mathrm{eV}
$$

sehingga

$$
M_{N 33} \geq \frac{100^{2}}{2 \times 10^{-12}} \mathrm{GeV} \geq 10^{15} \mathrm{GeV}
$$

dan bentuk matriks dominan $M_{N}$

$$
M_{N} \approx\left(\begin{array}{lll}
0 & 0 & 0 \\
0 & 0 & 0 \\
0 & 0 & 1
\end{array}\right)
$$

\section{Bauran Bimaksimal}

Jika $\sin \theta \approx 1 / \sqrt{2}$ maka kita mempunyai dua bauran maksimal yaitu $U(23)$ dan $U(12)$. Untuk kasus ini kita dapat membagi dalam tiga subkasus yaitu $\left|m_{2}\right|>>\left|m_{1}\right|, m_{2} \approx m_{1}$ dan $m_{2} \approx-m_{1}$.

1. Untuk kasus $\left|m_{2}\right|>>\left|m_{1}\right|$, kita dapatkan

$$
\begin{aligned}
& \alpha=\alpha^{\prime}=\frac{m_{2}}{2} \\
& \beta=\frac{\epsilon m_{3}+m_{2} / 2}{\sqrt{2}} \\
& \gamma=\frac{\epsilon m_{3}-m_{2} / 2}{\sqrt{2}}
\end{aligned}
$$

Dengan demikian invers matriks massa dengan $2 \epsilon m_{3}<<$ $\left|m_{2}\right|$ didapatkan

$$
M_{\nu}^{-1} \approx \frac{1}{4 m_{1}}\left(\begin{array}{ccc}
2 & -\sqrt{2} & \sqrt{2} \\
-\sqrt{2} & 1 & -1 \\
\sqrt{2} & -1 & 1
\end{array}\right)
$$

Lebih lanjut didapatkan

$$
M_{N 33} \approx \frac{k^{2} m_{\tau}^{2}}{4 m_{1}}
$$

Untuk sudut baur besar LMA,

$$
m_{2}=\sqrt{\Delta m_{\odot}^{2} \approx 10^{-5} e V^{2}} \approx 10^{-3} e V>>m_{1}
$$

Misalkan, ambil orde $m_{1}$ lebih kecil tetapi paling dekat dengan $m_{2}$

$$
m_{1} \leq 10^{-4} \mathrm{eV}
$$

maka

$$
M_{N 33} \geq 10^{16} \mathrm{GeV} \quad(L M A)
$$

yakni skala GUT atau skala unifikasi. Sedangkan untuk osilasi vakum (VO)

$$
m_{2}=\approx 10^{-5} \mathrm{eV}>>m_{1}
$$

Ambil $m_{1} \leq 10^{-6} \mathrm{eV}$ maka

$$
M_{N 33} \geq 10^{18} \mathrm{GeV} \quad(\mathrm{VO})
$$

yaitu skala Planck.

2. Untuk kasus $m_{2} \approx m_{1}$ maka

$$
\alpha^{\prime}=m_{2}, \quad \beta=\gamma=\frac{\epsilon m_{3}}{2}
$$

dan invers matriks massa neutrino

$$
M_{\nu}^{-1} \approx \frac{1}{-\Sigma}\left(\begin{array}{ccc}
m_{2} m_{3} & 0 & 0 \\
0 & y & -y \\
0 & -y & y
\end{array}\right)
$$

dengan $y=\frac{m_{3}}{2}\left(m_{2}-\epsilon^{2} m_{3}\right)$. Matriks massa neutrino ini dengan $m_{2}>>\epsilon^{2} m_{3}$ memberi suku $M_{N 33}$ Majorana masif

$$
M_{N 33} \approx \frac{k^{2} m_{\tau}^{2}}{2 m_{2}}
$$

dan $M_{N 33} \geq 10^{15} \mathrm{GeV}$ baik untuk LMA maupun VO. Matriks $M_{N}$ juga hirarkis dengan bentuk dominan sebagaimana bentuk (36).

3. Kasus $m_{2} \approx-m_{1}$ memberi

$$
\begin{aligned}
\alpha & =\alpha^{\prime}=0 \\
\beta & =\frac{\epsilon m_{3}+m_{2}}{\sqrt{2}} \\
\gamma & =\frac{\epsilon m_{3}-m_{2}}{\sqrt{2}}
\end{aligned}
$$

Selanjutnya, asumsi $\epsilon m_{3}<<m_{2}$ memberikan

$$
\beta=-\gamma=\frac{m_{2}}{\sqrt{2}}
$$


sehingga

$$
M_{\nu}^{-1} \approx \frac{1}{-\Sigma}\left(\begin{array}{ccc}
0 & -\frac{m_{2} m_{3}}{\sqrt{2}} & \frac{m_{2} m_{3}}{\sqrt{2}} \\
-\frac{m_{2} m_{3}}{\sqrt{2}} & -\frac{m_{2}^{2}}{2} & -\frac{m_{2}^{2}}{2} \\
\frac{m_{2} m_{3}}{\sqrt{2}} & -\frac{m_{2}^{2}}{2} & -\frac{m_{2}^{2}}{2}
\end{array}\right)
$$

Invers matriks massa neutrino tersebut memberikan elemen matriks massa neutrino efektif

$$
M_{N 13} \approx \frac{k^{2} m_{u} m_{t}}{\sqrt{2} m_{2}}, M_{N 33} \approx \frac{k^{2} m_{t}^{2}}{2 m_{3}}
$$

Jika $m_{2} / m_{3} \approx m_{u} / m_{t}$ maka $M_{N 13} \approx M_{N 33}$ dan dekat dengan skala unifikasi.

Kasus menarik $\delta \approx 0$ yang mungkin terjadi jika $m_{2}<0$ dan $\left|m_{2}\right| \approx \epsilon m_{3}$ sehingga $\gamma \approx-\sqrt{2} m_{2}$, maka

$$
M_{\nu}^{-1} \approx \frac{1}{-\Sigma}\left(\begin{array}{ccc}
0 & -\frac{m_{2} m_{3}}{\sqrt{2}} & \frac{m_{2} m_{3}}{\sqrt{2}} \\
-\frac{m_{2} m_{3}}{\sqrt{2}} & 2 \epsilon m_{2} m_{3} & 0 \\
\frac{m_{2} m_{3}}{\sqrt{2}} & 0 & 0
\end{array}\right)
$$

Matriks invers ini dan hubungan (3) memberikan matriks massa neutrino Majorana

$$
M_{N} \approx \frac{k^{2}}{m_{2}}\left(\begin{array}{ccc}
0 & -\frac{m_{u} m_{c}}{\sqrt{2}} & \frac{m_{u} m_{t}}{\sqrt{2}} \\
-\frac{m_{u} m_{c}}{\sqrt{2}} & 2 \epsilon m_{c}^{2} & 0 \\
\frac{m_{u} m_{t}}{\sqrt{2}} & 0 & 0
\end{array}\right)
$$

yang memberi bentuk dominan

$$
M_{N} \approx\left(\begin{array}{lll}
0 & 0 & 1 \\
0 & 0 & 0 \\
1 & 0 & 0
\end{array}\right)
$$

Massa terberat

$$
M_{N 13} \approx \frac{k^{2}}{\sqrt{2}} \frac{m_{u} m_{t}}{m_{2}}
$$

untuk $m_{2} \leq 10^{-3} \mathrm{eV}$ maka

$$
M_{N 13} \geq \frac{(0,001)(100)}{10^{-12}} \mathrm{GeV} \geq 10^{11} \mathrm{GeV}
$$

yakni skala antara (intermediate scale) sebagaimana diperoleh sebelumnya [13].

Untuk $\gamma \approx 0$ dan $\beta \approx \sqrt{2} m_{2} \approx \sqrt{2} \epsilon m_{3}$ diperoleh

$$
M_{\nu}^{-1} \approx \frac{1}{-\Sigma}\left(\begin{array}{ccc}
0 & -\frac{m_{2} m_{3}}{\sqrt{2}} & \frac{m_{2} m_{3}}{\sqrt{2}} \\
-\frac{m_{2} m_{3}}{\sqrt{2}} & 0 & 0 \\
\frac{m_{2} m_{3}}{\sqrt{2}} & 0 & -2 \epsilon m_{2} m_{3}
\end{array}\right) \text { (57) }
$$

dan

$$
M_{N 13} \approx \frac{k^{2} m_{u} m_{t}}{\sqrt{2} m_{2}}, M_{N 33} \approx \frac{2 k^{2} \epsilon m_{t}^{2}}{m_{2}}
$$

Jika $\epsilon \approx m_{u} / m_{t}$ maka $M_{N 13} \approx M_{N 33}$ dan dekat dengan skala unifikasi.

\section{B. Hirarki Terbalik}

Sekarang kita tinjau bila $\left|m_{1}\right| \approx\left|m_{2}\right|>>m_{3}$. Dalam kasus ini berlaku

$$
\Delta m_{a t m}^{2}=m_{2}^{2}-m_{3}^{2} \approx m_{2}^{2}
$$

Kondisi ini membuat elemen (20) menjadi

$$
\begin{aligned}
\alpha & =\cos ^{2} \theta m_{1}+\sin ^{2} \theta m_{2} \\
\alpha^{\prime} & =\sin ^{2} \theta m_{1}+\cos ^{2} \theta m_{2} \\
\beta & =\frac{-\epsilon \alpha+\sin \theta \cos \theta\left(m_{2}-m_{1}\right)}{\sqrt{2}} \\
\gamma & =\frac{-\epsilon \alpha-\sin \theta \cos \theta\left(m_{2}-m_{1}\right)}{\sqrt{2}} \\
\rho & =\rho^{\prime}=-\sigma \approx \frac{\alpha^{\prime}}{2}
\end{aligned}
$$

Dengan demikian matriks massa neutrino efektif diberikan oleh

$$
M_{\nu}=\left(\begin{array}{ccc}
\alpha & \beta & \gamma \\
\beta & \frac{\alpha^{\prime}}{2} & -\frac{\alpha^{\prime}}{2} \\
\gamma & -\frac{\alpha^{\prime}}{2} & \frac{\alpha^{2}}{2}
\end{array}\right)
$$

Menggunakan elemen-elemen (60) diperoleh $\left(M_{\nu}^{-1}\right)_{11}=$ 0 . Elemen ini memang sangat kecil tetapi tidak perlu nol dan dipenuhi bila digunakan

$$
\begin{aligned}
\sigma & =\frac{m_{3}-\alpha^{\prime}}{2} \\
\rho & =\rho^{\prime}=\frac{m_{3}+\alpha^{\prime}}{2}
\end{aligned}
$$

dan didapatkan $\left(M_{\nu}^{-1}\right)_{11}=\frac{m_{3} \alpha^{\prime}}{-\Sigma}$. Secara lengkap, $\left(M_{\nu}^{-1}\right)$ diberikan oleh

$$
M_{\nu}^{-1} \approx \frac{1}{-\Sigma}\left(\begin{array}{ccc}
m_{3} \alpha^{\prime} & -\frac{\alpha^{\prime}}{2}(\beta+\gamma) & -\frac{\alpha^{\prime}}{2}(\beta+\gamma) \\
-\frac{\alpha^{\prime}}{2}(\beta+\gamma) & \frac{\alpha \alpha^{\prime}}{2}-\gamma^{2} & \beta \gamma+\frac{\alpha \alpha^{\prime}}{2} \\
-\frac{\alpha^{\prime}}{2}(\beta+\gamma) & \beta \gamma+\frac{\alpha \alpha^{\prime}}{2} & \frac{\alpha \alpha^{\prime}}{2}-\beta^{2}
\end{array}\right)
$$


Selanjutnya kita selidiki untuk kasus demi kasus.

\section{Bauran Maksimal Tunggal}

Pertama ambil kasus $\theta \approx 0$ maka besaran-besaran (60) menjadi

$$
\begin{aligned}
& \alpha=m_{1} \alpha^{\prime}=m_{2} \\
& \beta=\gamma=\frac{-\epsilon m_{1}}{\sqrt{2}}
\end{aligned}
$$

Matriks massa neutrino efektif diberikan oleh

$$
M_{\nu} \approx\left(\begin{array}{ccc}
m_{2} & -\frac{\epsilon m_{2}}{\sqrt{2}} & -\frac{\epsilon m_{2}}{\sqrt{2}} \\
-\frac{\epsilon m_{2}}{\sqrt{2}} & \frac{m_{2}}{2} & -\frac{m_{2}}{2} \\
-\frac{\epsilon m_{2}}{\sqrt{2}} & -\frac{m_{2}}{2} & \frac{m_{2}}{2}
\end{array}\right)
$$

Bentuk dominannya diberikan oleh

$$
M_{\nu} \approx\left(\begin{array}{lll}
1 & 0 & 0 \\
0 & 1 & 1 \\
0 & 1 & 1
\end{array}\right)
$$

Matriks inversnya diberikan oleh

$$
M_{\nu}^{-1} \approx \frac{1}{m_{3}}\left(\begin{array}{ccc}
\frac{m_{3}}{m_{2}} & \frac{\epsilon}{\sqrt{2}} & \frac{\epsilon}{\sqrt{2}} \\
\frac{\epsilon}{\sqrt{2}} & \frac{1}{2} & \frac{1}{2} \\
\frac{\epsilon}{\sqrt{2}} & \frac{1}{2} & \frac{1}{2}
\end{array}\right)
$$

Matriks invers ini memberi bentuk dominan bagi $M_{N}$ sebagaimana bentuk (36) dengan massa terbesar

$$
M_{N 33} \approx \frac{2 k^{2} m_{t}^{2}}{2 m_{3}}
$$

dan pada skala unifikasi atau di atasnya.

\section{Bauran Bimaksimal}

Untuk sudut $\theta \approx \pi / 4$ terdapat dua subkasus yaitu $m_{2} \approx m_{1}$ dan $m_{2} \approx-m_{1}$.

1) Untuk $m_{2} \approx m_{1}$ maka

$$
\begin{aligned}
& \alpha \approx \alpha^{\prime} \approx m_{1} \\
& \beta \approx \gamma \approx-\frac{\epsilon m_{2}}{\sqrt{2}}
\end{aligned}
$$

dan matriks massa neutrino efektif

$$
M_{\nu} \approx\left(\begin{array}{ccc}
m_{2} & -\frac{\epsilon m_{2}}{\sqrt{2}} & -\frac{\epsilon m_{2}}{\sqrt{2}} \\
-\frac{\epsilon m_{2}}{\sqrt{2}} & \frac{m_{2}}{2} & -\frac{m_{2}}{2} \\
-\frac{\epsilon m_{2}}{\sqrt{2}} & -\frac{m_{2}}{2} & \frac{m_{2}}{2}
\end{array}\right)
$$

Tampak memberi hasil yang sama dengan kasus sudut kecil $\theta \approx 0$ untuk hirarki terbalik.
2) Untuk $m_{2} \approx-m_{1}$ diperoleh

$$
\begin{aligned}
& \alpha \approx \alpha^{\prime} \approx 0 \\
& \beta \approx-\gamma \approx-\frac{m_{2}}{\sqrt{2}}
\end{aligned}
$$

Karena itu, matriks massa neutrino efektif

$$
M_{\nu} \approx\left(\begin{array}{ccc}
0 & \frac{m_{2}}{\sqrt{2}} & -\frac{m_{2}}{\sqrt{2}} \\
\frac{m_{2}}{\sqrt{2}} & 0 & 0 \\
-\frac{m_{2}}{\sqrt{2}} & 0 & 0
\end{array}\right)
$$

dan bentuk dominannya

$$
M_{\nu} \approx\left(\begin{array}{lll}
0 & 1 & 1 \\
1 & 0 & 0 \\
1 & 0 & 0
\end{array}\right)
$$

Matriks invers

$$
M_{\nu}^{-1} \approx \frac{1}{-2 m_{3}}\left(\begin{array}{lll}
0 & 0 & 0 \\
0 & 1 & 1 \\
0 & 1 & 1
\end{array}\right)
$$

yang memberi massa terbesar

$$
M_{N 33} \approx \frac{k^{2} m_{t}^{2}}{2 m_{3}}
$$

dengan skala unifikasi atau di atasnya.

\section{DISKUSI DAN SIMPULAN}

Pada pembahasan ini hanya ditinjau matriks bauran riel atau tanpa sudut fasa. Sudut fasa ini dapat menjadi sumber informasi bagi terjadinya alam semesta taksimetri saat ini, sebagai contoh [14]. Tanda dari $m_{i}$ terkait dengan paritas CP dari neutrino sedangkan massa fisis $\left|m_{i}\right|[15]$.

Analisa memberikan dua bentuk dominan $M_{N}$ yaitu bentuk diagonal (36) dan off-diagonal (54). Bentuk diagonal umumnya berskala unifikasi kecuali pada kasus osilasi vakum yang berskala di atas unifikasi menuju skala Planck. Sedangkan bentuk off-diagonal pada skala antara.

\section{Ucapan Terima Kasih}

Penulis sampaikan terimakasih kepada Anwari Fundamental Science Foundation (AFSiF) yang mendukung penelitian ini. 
[1] Y. Fukuda $d k k$., Phys.Rev.Lett. 81, 1158(1998); M.H. Ahn $d k k$.,Phys.Rev.Lett. 90, 041801(2003); Q.R. Ahmad $d k k$., Phys.Rev.Lett. 89, 011301; 011302(2002); K.Eguchi $d k k$., Phys.Rev.Lett. 90, 021802(2003).

[2] M. Gell-Mann, P.Ramond and R. Slansky, in Supergravity, eds. P. van Nieuwenhuizen and D. Freedman (North Hollad, Amsterdam, 1979); T.Yanagida, in Proceedings of the Workshop on Unified Theories and Baryon Number in the Universe, eds. O.Sawada and A. Sugamoto (KEK, Tsukuba, 1979).

[3] H. Nishiura, K.Matsuda and T. Fukuyama, Phys. Rev. D60 013006 (1999); H. Fritzsch and Z. Xing, Nucl. Phys. B556 49 (1999).

[4] P. Ramond, R.G.Roberts and G.G. Ross, Nucl.Phys. B406 19 (1993).

[5] H. Arason, D.J. Castano, E.J. Piard and P. Ramond, Phys.Rev. D47 232 (1993).

[6] H. Fusaoka and Y. Koide, Phys. Rev. D57, 3986 (1998).
[7] B. Pontecorvo, Zh. Eksp. Teor. Fiz. 33549 (1957); Z. Maki, M. Nakagawa, and S. Sakata, Prog. Theor. Phys. 28, 870 (1962).

[8] J.N. Bahcal, P.I. Krastev and A. Yu. Smirnov, Phys. Rev. D58, 096016 (1998); D60, 093001 (1999).

[9] E. Kh. Akhmedov, Phys. Lett B467, 95 (1999).

[10] M.Appolonio dkk. [CHOOZ Collab.], Phys.Lett. B420,397(1998).

[11] Y. Fukuda $d k k .$, Phys.Rev.Lett. 81, 1562(1998).

[12] D. Falcone, hep-ph/0002242.

[13] M. Jezabek and Y. sumino, Phys.Lett. B440, 327 (1998); B. Stech, Phys.Lett. B465, 219 (1999).

[14] T. Endoh, T. Morozumi, T. Onogi, and A. Purwanto, Phys. Rev. D64, (2001)013006; T. Endoh, T. Morozumi, and A. Purwanto, Nucl.Phys.Proc.Suppl. B111, 299(2001).

[15] S.M.Bilenky, C. Giunti and W. Grimus, Prog. Part. Nucl. Phys. 431 (1999). 